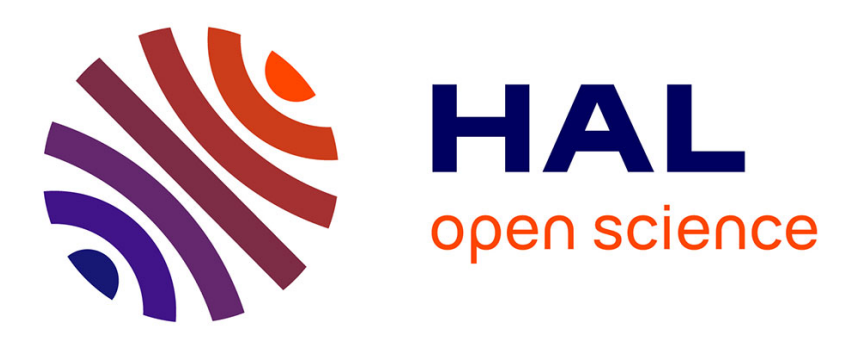

\title{
Optimal drift region for diamond power devices
}

Gauthier Chicot, David Eon, Nicolas C. Rouger

\section{To cite this version:}

Gauthier Chicot, David Eon, Nicolas C. Rouger. Optimal drift region for diamond power devices. Diamond and Related Materials, 2016, 69, pp.68-73. 10.1016/j.diamond.2016.07.006 . hal-01990401

\section{HAL Id: hal-01990401 https://hal.science/hal-01990401}

Submitted on 13 Jul 2021

HAL is a multi-disciplinary open access archive for the deposit and dissemination of scientific research documents, whether they are published or not. The documents may come from teaching and research institutions in France or abroad, or from public or private research centers.
L'archive ouverte pluridisciplinaire HAL, est destinée au dépôt et à la diffusion de documents scientifiques de niveau recherche, publiés ou non, émanant des établissements d'enseignement et de recherche français ou étrangers, des laboratoires publics ou privés. 


\title{
Optimal drift region for diamond power devices
}

\author{
Gauthier CHICOT ${ }^{1,2}$, David EON ${ }^{1,3}$, Nicolas ROUGER ${ }^{1,2}$ \\ ${ }^{1}$ Univ. Grenoble Alpes, F-38000 Grenoble, France \\ ${ }^{2}$ CNRS, G2ELab, F-38000 Grenoble, France \\ ${ }^{3}$ CNRS, Institut Néel, F-38000 Grenoble, France
}

\begin{abstract}
In power devices such as Schottky Barrier Diodes or Field Effect Transistors, the breakdown voltage is linked to the design of the drift layer but also to the physical properties of the material used. Diamond, with its high critical electric field due to its large band gap, opens the way to power components able to withstand very high voltage with outstanding figures of merit. Nevertheless, a particular attention has to be paid to the design of the drift layer to take benefit of these outstanding properties. Indeed, the drift region thickness, doping level and consequently the punch through or non-punch through designs must be well designed to reach the desired breakdown value and to minimize the ON state resistance at the same time. Here, a focus on the optimization of the specific ON state resistance as function of the breakdown voltage figure of merit has been carried out, while optimizing the drift layer and calculating the specific ON state resistance of unipolar high voltage diamond power devices. Based on the ionization integral calculation with impact ionization coefficients adapted to diamond, we performed an accurate analysis to find the best punch through design of the drift layer offering the lowest ON state resistance at a given breakdown voltage value. This theoretical study has been first applied in a one dimensional discrete approach of the breakdown voltage. An additional 2D cylindrical coordinate analysis was performed to quantify the radius effect on the breakdown voltage value, and to compare the $2 \mathrm{D}$ breakdown voltage with the $1 \mathrm{D}$ breakdown voltage, for different drift region designs. These results offer preliminary design rules to fabricate more efficient unipolar diamond power devices. At the material level, this analysis also points out that thicknesses and doping levels required to achieve such structures are quite challenging for crystal growth in the context of high voltage power devices.
\end{abstract}

\section{Key word}

Numerical design of experiences, drift layer, breakdown voltage, punch through design, diamond, unipolar component, figure of merit.

\section{Introduction}

As the demand in high power and high frequency electronics keep increasing, standard semiconductors show their limits. Indeed, components able to operate at high voltage, high frequency and high temperature are crucial to go beyond the classical design trade off in power devices. Diamond, thanks to its outstanding properties, is the ultimate semiconductor to meet these requirements [1]. In power electronics, Schottky Barrier Diode (SBD) rectifier and Field Effect Transistor (FET) switch are two complementary devices in the commutation cell of 
power converters. On one hand, diamond SBDs able to withstand up to $10 \mathrm{kV}$ have been already reported [2-6] . On the other hand, diamond FETs are under investigation through MESFET [7-10] and MOSFET with hydrogen terminated surface [11-14] while recent works on oxygen terminated diamond MOS capacitors [15-17] suggests that normally OFF diamond MOSFET are also worth considering.

In both devices, a specific thick and doped layer must be validated to withstand high voltage and it is crucial to optimize the design structure of unipolar components to take advantage of diamond material properties to fabricate devices with the best possible performances. On one hand, the ON state is directly linked to the ability of the device to let the current flow through it, usually figured as the specific ON state resistance $\left(R_{o n} . S\right)$. On the other hand, the OFF state performance is depicted by the Breakdown Voltage (BV) which represents the ability to support the blocking voltage. These two physical quantities are directly linked to the properties and the design of the drift layer. Sizing this layer to withstand high voltage will result in increasing its resistivity and will therefore directly reduce the ON state performances of the device. More particularly, for a fixed breakdown voltage, only one drift layer design (doping level and thickness) will lead to the lowest $R_{o n} . S$ possible. It is a matter of importance to find this optimum design for the drift layer. Although such a study has been widely conducted for various semiconductors based on well identified impact ionization coefficients [18,19], optimal drift layer design rules for diamond is still not available in the community.

Such work requires having good theoretical knowledge of the diamond material to perform calculation and simulation prior to fabrication of devices. Diamond physical specific properties models such as incomplete ionization, empirical activation energy and empirical mobility models have been already proposed and are still improved day by day. However, there is still no experimental report on the impact ionization coefficient needed to simulate the breakdown voltage of diamond devices. Realistic values of these parameters extrapolated from silicon adapted to experimental measurements on diamond devices [20] and others from ab-initio calculations [21] have been recently proposed. These values are in most of the case in good agreement with performances reported for several devices in literature. However, previous diamond power devices with high voltage $(>1 \mathrm{kV})$ do not have efficient and reliable Junction Termination Extension (JTE) [22,23], questioning the experimental data and consequently fitted impact ionization coefficients. More works are required to reduce peaks in the 3D electric field distribution, and the extracted impact ionization coefficients will be more likely updated in the future. Using the realistic model proposed by Hiraiwa et al. [12], the optimal drift layer parameters were considered as a function of the targeted breakdown voltage. For the breakdown voltage analysis, avalanche breakdown has been considered, using a one dimensional discrete approach [24]. Similar analysis based on other methods have been reported on diamond $[20,23,25]$. In this paper, a complementary study of the reciprocal punch through (PT) factor (ratio of the drift layer thickness in punch through design to the one in non-punch through design) of unipolar diamond power components as function of the breakdown voltage is performed. Therefore, the optimal reciprocal PT factor for which the specific ON state resistance of the drift layer is minimized can be determined. Although well known in silicon [26] and silicon carbide [18], such studies have never been performed on diamond with realistic and up to date impact ionization coefficients. A cylindrical 2D approach was also led in order to see the influence of the radius on the reached $\mathrm{BV}$ compared to the theoretical $1 \mathrm{D}$ case.

In a first part the numerical models used in this work and the simulated structure will be briefly described. Then, the corresponding results in 1D and 2D will be exposed. Before concluding, we will show how 
performances of devices from literature could have been improved by taking into account the design rules proposed in this work.

\section{Materials and Methods}

In order to calculate accurately the specific ON state resistance, two models specific to diamond must be used: the incomplete ionization model and the empirical mobility model [27] which are described in details in Maréchal et al. work [28]. These two models take into account the temperature dependence as well as the boron dopant activation energy dependence to the doping level [29].

In order to compute the $\mathrm{BV}$ of the considered drift layer, one needs to know the avalanche impact ionization coefficient for holes and electrons, $\alpha_{p}$ and $\alpha_{n}$ given by: $\alpha_{p}=a_{p} \exp \left(\frac{-b_{p}}{|E|}\right), \alpha_{n}=a_{n} \exp \left(\frac{-b_{n}}{|E|}\right)$ (Eq.1) where $|E|$ is the electric field in the junction, $a_{p}\left(a_{n}\right)$ and $b_{p}\left(b_{n}\right)$ are the coefficients for holes (electrons) depending on the impact ionization models. These coefficients are used to calculate the multiplication coefficients $\left(M_{n}, M_{p}\right)$, respectively for holes and electrons that we will use as a criterion to determine the breakdown voltage value. In this work, coefficient proposed recently by Hiraiwa et al. [20] are used: $a_{n}=1.4 \times 10^{5} \mathrm{~cm}^{-1}, b_{n}=2.4 \times 10^{7} \mathrm{~V} / \mathrm{cm}$, $a_{p}=6.1 \times 10^{4} \mathrm{~cm}^{-1}, b_{p}=1.4 \times 10^{7} \mathrm{~V} / \mathrm{cm}$. These coefficients were adapted to performances of devices found in the literature and are up to date the more realistic ones. Accordingly to this modeling, the BV is supposedly related to an avalanche breakdown mechanism. In this study we do not take into account leakage current that can limit the BV value before reaching the avalanche phenomena.

All these models were implemented in the approach of avalanche breakdown calculation described in details in Rouger work [24]. This method was chosen for its accuracy (more than an analytical method which needs approximations) and its shorter CPU time compared to a finite element numerical method. Nevertheless, the analysis of the reciprocal punch through factor proposed in this work can be performed by following the methodology presented in this novel contribution with any BV calculation technique that one would like to use. In this work, we will assume that the avalanche breakdown occurs when the multiplication factor is greater than 200 (i.e. ionization integral above 0.995).

The simulated structure is composed of a stack of $n+$ type diamond, $p$ type diamond and $p+$ type diamond as schematized on Figure 1. The $n+$ type doping level was chosen in order that the space charge region extends mainly in the $p$ type drift layer as it is shown by the grey part on Figure 1.Therefore, with the method and models described above, we are able to calculate the breakdown voltage and the specific ON state resistance of $n+/ p / p+$ structure with several drift layer doping levels and thicknesses. As the drift layer will represent the main contribution to the specific ON state resistance (particularly for high voltage designed structures), only its contribution will be considered in the following. Nevertheless, one has to consider the contacts and others layers contribution to accurately evaluate the total $\mathrm{ON}$ state resistance of a device [4]. Contrary to most of the $\mathrm{Si}$ components where the drift layer is made of $n$ type material, in diamond it is more beneficial to design the component with a $p$ type drift layer. In fact, the $\mathrm{ON}$ state resistance of $p$ type diamond material is lower than the one of $n$ type material due to a higher mobility $\left(2000 \mathrm{~cm}^{2} / \mathrm{V}\right.$.s for holes versus $1000 \mathrm{~cm}^{2} / \mathrm{V}$.s for electrons) and a lower activation energy of dopants $(0.38 \mathrm{eV}$ for Boron dopants versus $0.57 \mathrm{eV}$ for Phosphorous and $1.7 \mathrm{eV}$ for 
Nitrogen dopants), leading to a larger number of ionized acceptors than donors for the same doping level at a given temperature.
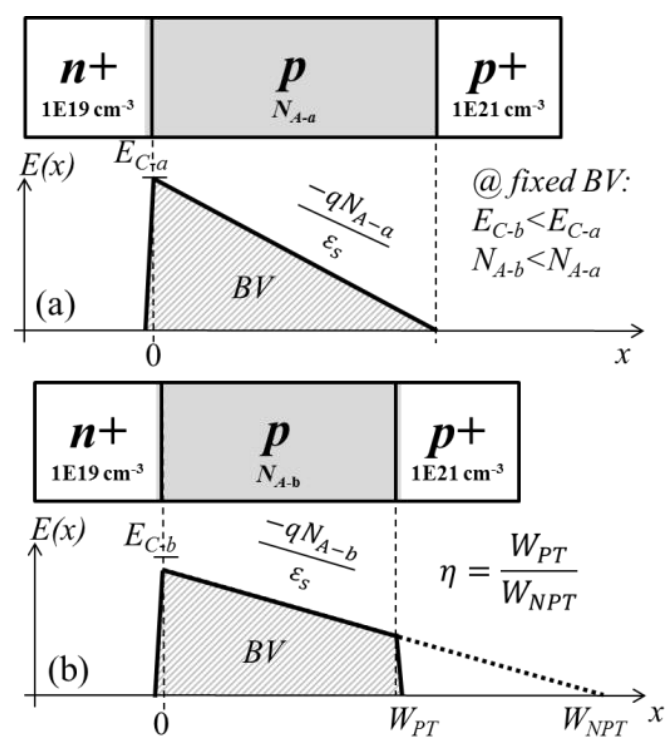

Figure 1 : Structure and electric field profile in (a) non-punch through and (b) punch through design considered in this work.

\section{Optimization of the drift layer}

The breakdown voltage as function of the drift layer doping level is plotted on Figure 2 for different cases, similar to [23,25] but based on updated impact ionization coefficients. If labeled with a length indication this refers to the thickness of the drift layer in PT design, which will be denoted as $W_{P T}$ in the following. In this PT case, which corresponds to Figure 1 (b), the space charge region extends through the $p$ type layer and this extension is stopped by the $p+$ type layer, meaning that the entire $p$ type layer contributes to support the blocking voltage. On the contrary in Non-Punch Through (NPT) case (Figure 1 (a)) only a part of the $p$ type layer is depleted and withstand the total voltage at avalanche breakdown. It has to be noticed that for the same BV value, the thickness and the doping of the drift layer are lower in PT than NPT. The critical field is also lower in PT than in NPT (i.e. $\mathrm{E}_{\mathrm{C}-\mathrm{b}}<\mathrm{E}_{\mathrm{C}-\mathrm{a}}$ in Figure 1). The starting point of our work was to compute the BV values as function of the doping values for several designs, either NPT or PT as plotted on Figure 2. These results are very similar quantitatively to those obtained by Hiraiwa et al. [20] (indeed, the impact ionization coefficients are taken from this work), thus validating our discrete approach. Similarly to Hiraiwa et al. work, experimental data points from literature $[2,5,6,30-34]$ are also added on the graph reinforcing the validity of our calculations. 


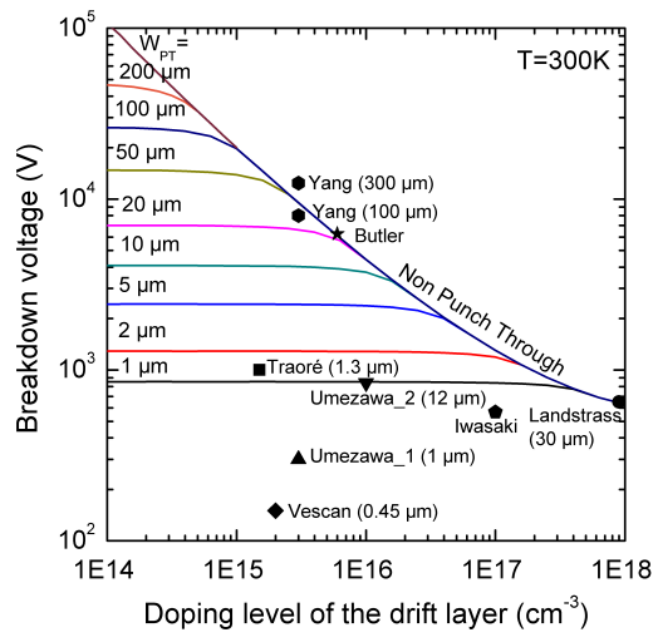

Figure 2: Breakdown voltage of a $n+/ p / p+$ stack versus doping and thickness of the drift layer. Plots are labeled with the corresponding thickness of the drift layer in punch through design while non-punch through design is indicated on the corresponding one. Experimental data points are from ref. [2,5,6,30-34], drift layer lengths are reported when available. Such a plot has already been published in ref. [20] with very similar values, thus validating our approach.

Figure 3 represents the $R_{o n} . S$ versus doping level of the different configurations of Figure 2. These two sets of data allow to plot $R_{o n} . S(B V)$ figures of merits as it has already been shown in ref. [1,4]. In this work, we focus on the optimization of the $R_{o n} S(B V)$ figure of merit while sizing the drift layer thickness and determining its doping level value. In other terms, we are interested in finding the optimum PT design which allows a given blocking voltage value while minimizing $R_{o n} . S$ compared to NPT design. Our method which is described in Figure 4 consists in generating sets of data similar to that plotted in Figure 2 and Figure 3, but with a more detailed sampling in term of drift layer thickness in PT design. The first step is to find the values of $W_{P T}$ and doping values that can allow reaching the chosen breakdown voltage. Then, one should find the corresponding specific on-state resistance for each of these PT designs. The third step is to compute the corresponding $W_{N P T}$ for all the doping values of the PT designs considered in the first and second steps. Finally, it is then possible to plot the specific ON state resistance as function of the reciprocal punch through factor $\eta=W_{P T} / W_{N P T}$ as it has been done on Figure 5 for different breakdown voltage values. The value of $\eta$ corresponding to the minimum of the specific ON state resistance for a fixed breakdown voltage is called the optimal reciprocal punch through coefficient and is denoted as $\eta_{\text {opt }}$. The optimum reciprocal PT coefficient is around 0.7 for the four BV values investigated $(1 \mathrm{kV}, 3 \mathrm{kV}, 6.5 \mathrm{kV}$ and $10 \mathrm{kV}$ ). For some $\eta$ values, the corresponding $W_{P T}$, drift layer doping level as well as $R_{o n .} S$ values are reported on Figure 5. In addition to $300 \mathrm{~K}$ plot, it has been chosen to plot these curves also at $500 \mathrm{~K}$. Indeed, diamond performances are not very competitive with silicon carbide at $300 \mathrm{~K}$ contrary to $500 \mathrm{~K}$ where the specific ON state resistance of diamond is greatly improved because of a much larger number of Boron dopants being ionized at this temperature. Please note that the set of impact ionization coefficients used in our work were determined at $300 \mathrm{~K}$ in Ref. [1]. Therefore, when computing $R_{o n} S(B V)$ figure of merit at $500 \mathrm{~K}$ only the influence of the temperature on $R_{o n} S$ was considered. For a more accurate and realistic analysis temperature dependence of impact ionization coefficients must be further provided [35,36]. Anyway, as soon as a temperature dependence model will be proposed, the approach described in this paper could be led again with the new set of coefficients to update the $R_{o n} S(B V)$ figure of merits at any temperature. 


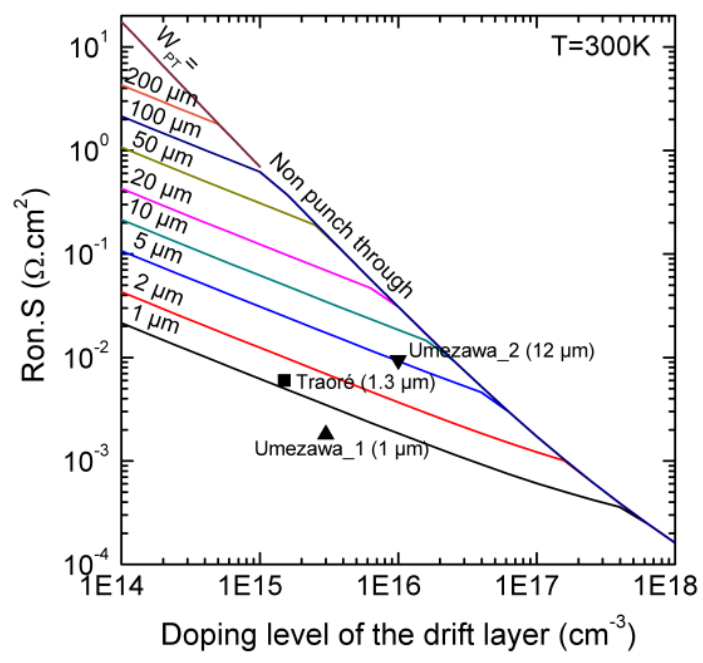

Figure 3: Specific ON state resistance versus doping level for different configurations of $n+/ p / p+$ stacks. Curves are labeled with the corresponding thickness of the drift layer in punch through designs while non punch through design is indicated on the corresponding one. Experimental data points are from ref. [2,32], drift layer lengths are reported in brackets. One has to note that the Breakdown Voltage is not constant in this plot.
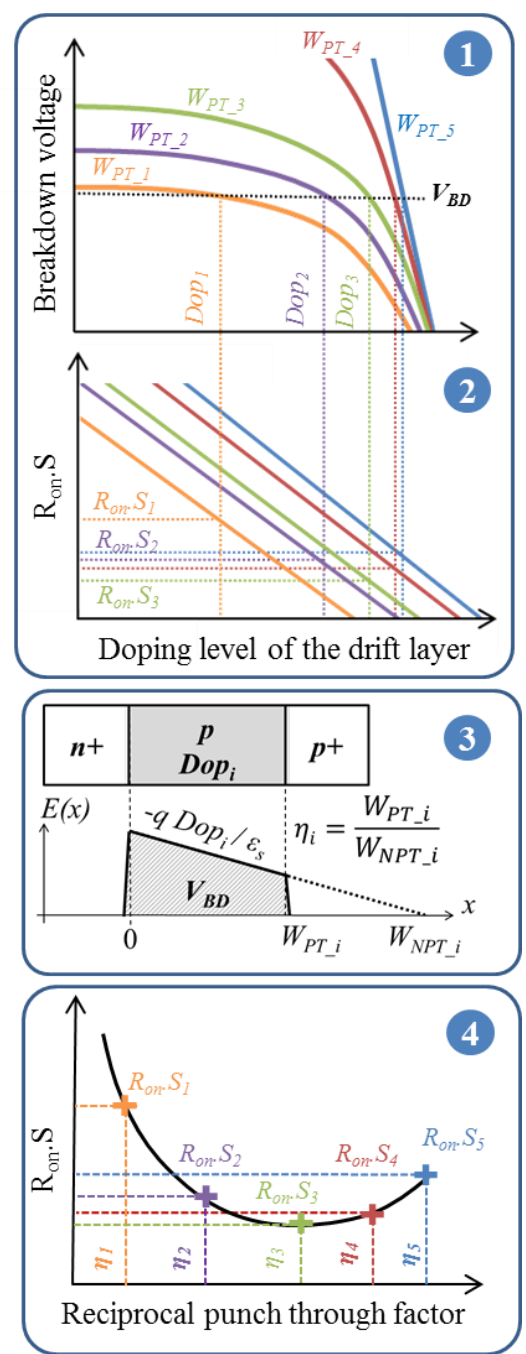

Figure 4: Method used to find the optimum reciprocal punch through coefficient at a given breakdown voltage value $\mathrm{V}_{B D}$. 
As summarized in Table 1, designing the drift layer using the optimal PT design can allow a reduction of the ON state resistance up to $16 \%$ compared to NPT design. In addition of a $R_{o n} . S$ lowering, PT design can also be helpful from a technological point of view as it can allow growing thinner layers which in general contain fewer defects than thicker ones. As an example, a $10 \mathrm{kV}$ diamond unipolar device require a drift layer thickness of at least $47.9 \mu \mathrm{m}$ in NPT design. Considering the optimal punch through design will make it possible to reduce the specific ON state resistance but also to grow a drift layer of $37 \mu \mathrm{m}$ which is more than $20 \%$ thinner than in PT design. Even thinner layers can be used if a smaller reciprocal PT coefficient is chosen, but it will be at the expense of the specific ON state resistance increase. In fact, one must be aware that if choosing a too much low reciprocal PT factor (far lower than the optimum one), the ON state resistance can be highly increased (by more than $50 \%$ for $\eta=0.1$ as an example). As it will be seen in the following, using PT design can also be beneficial regarding $2 \mathrm{D}$ (or $3 \mathrm{D}$ ) impact on the reached $\mathrm{BV}$. In any case, reaching the optimum design will require a high accuracy on targeted doping level and thickness values during the growth of the drift layer. 

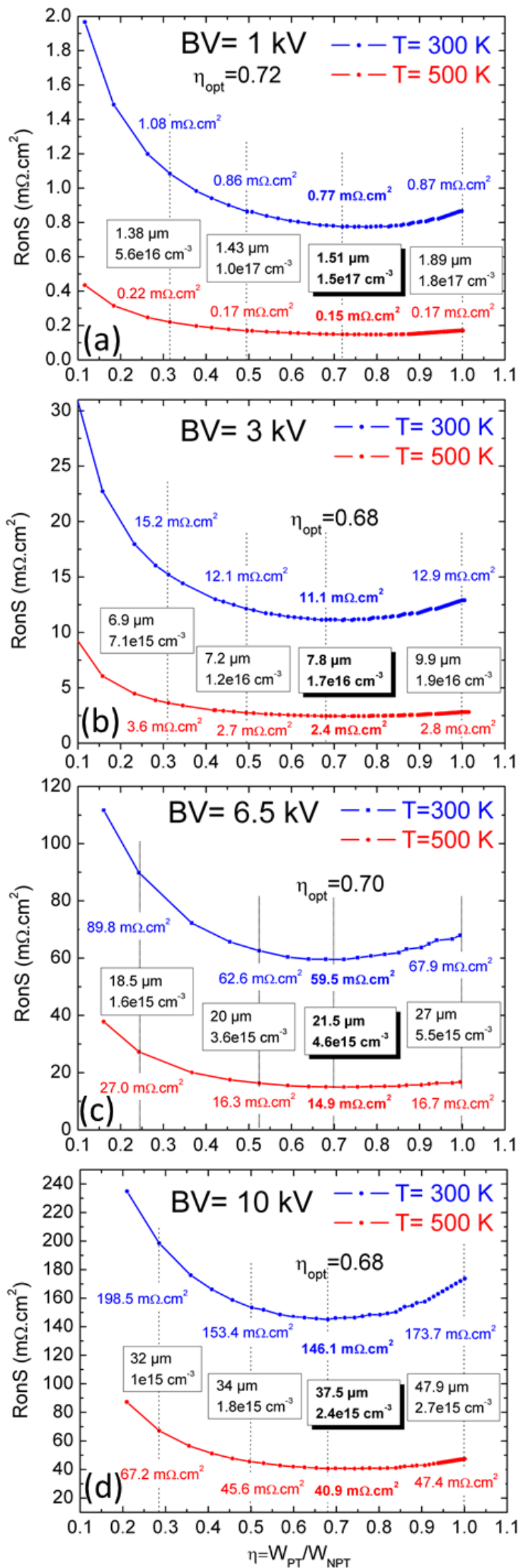

Figure 5: Specific ON state resistance versus reciprocal punch through factor at $300 \mathrm{~K}$ and $500 \mathrm{~K}$ for breakdown voltage of (a) $1 \mathrm{kV}$, (b) $3 \mathrm{kV}$, (c) $6.5 \mathrm{kV}$ and (d) $10 \mathrm{kV}$. 
Table 1: Optimum drift region design parameters (doping and drift region thickness) and the ON state resistance reduction by using the optimum punch through design $\mathbf{P T}_{\text {opt }}$ (for $\eta=\eta_{\text {opt }}$ ) compared to non-punch through PT one (for $\eta=1)$.

\begin{tabular}{ccccc}
\hline \hline Breakdown voltage & $1 \mathrm{kV}$ & $3 \mathrm{kV}$ & $6.5 \mathrm{kV}$ & $10 \mathrm{kV}$ \\
\hline Doping $\left(\eta_{\text {opt }}\right)[\mathrm{cm}-3]$ & $1.5 \times 10^{17}$ & $1.7 \times 10^{16}$ & $4.6 \times 10^{15}$ & $2.4 \times 10^{15}$ \\
\hline$W_{P T}\left(\eta_{\text {opt }}\right)[\mu \mathrm{m}]$ & 1.5 & 7.8 & 21.5 & 37.5 \\
\hline $\left.\begin{array}{c}1-R_{\text {on }} . S(\mathrm{PT} \\
\text { opt }\end{array}\right) / R_{o n} . S(\mathrm{NPT})$ & $11 \%$ & $14 \%$ & $12 \%$ & $16 \%$ \\
\hline$T=300 \mathrm{~K}$ & $11 \%$ & $14 \%$ & $11 \%$ & $16 \%$ \\
\hline $\left.\begin{array}{c}1-R_{o n} . S(\mathrm{PT} \\
\text { opt }\end{array}\right) / R_{o n} . S(\mathrm{NPT})$ & & & & \\
\hline \hline
\end{tabular}

\section{D cylindrical BV versus 1D BV}

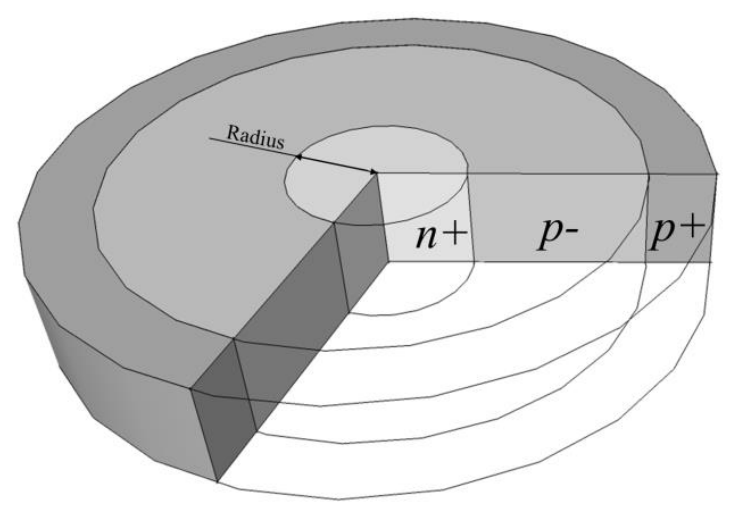

Figure 6: Scheme of the structure considered in 2D cylindrical coordinates.

1D breakdown is a first approach to evaluate the BV of a given drift layer but real devices will rarely reach the high voltage performances of a plane junction. JTE efficiency must be then optimized to reach $100 \%$ BV of the 1D plane junction, and diamond must follow recent developments in $\mathrm{GaN}$ [37] and $\mathrm{SiC}$ [38] with specific limitations related to diamond (e.g. implanted layers for guard rings are not possible). To investigate such a phenomenon, 2D cylindrical structures have been considered as depicted on Figure 6. The calculation method is derived from 1D one and is also described in details in ref [24]. Using this method it is possible to compare the breakdown voltage of a 1D structure with the same structure (i.e. doping level and thickness of the layers) in 2D cylindrical coordinates. 


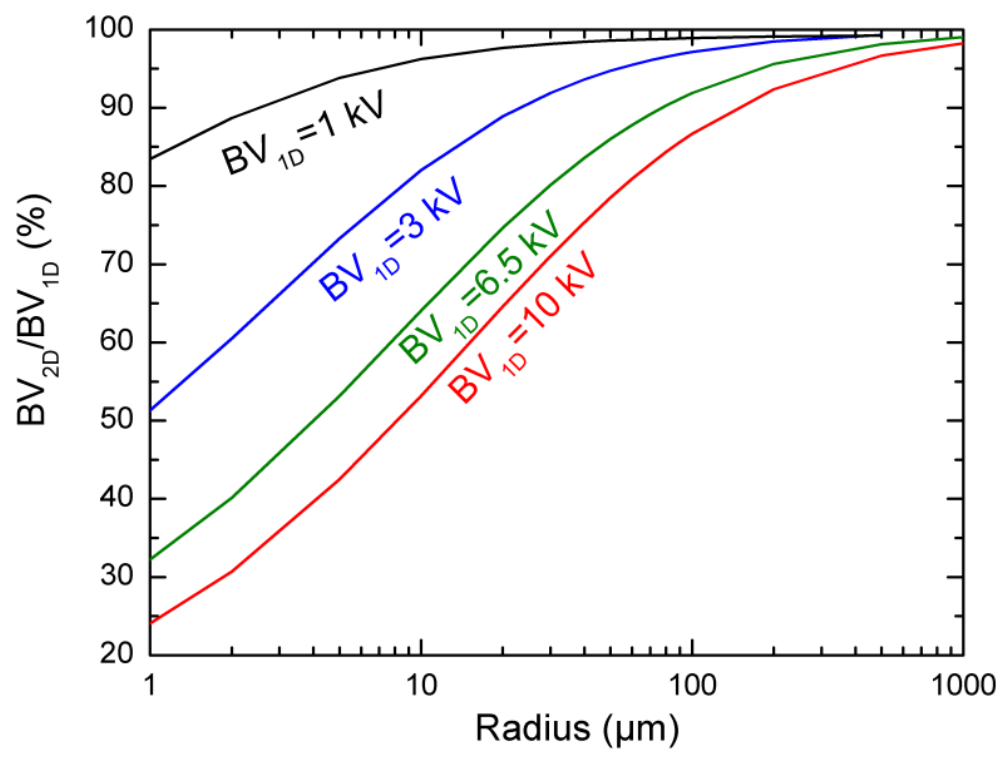

Figure 7: Normalized breakdown voltage (ratio of $2 \mathrm{D} \mathrm{BV}$ by $1 \mathrm{D} \mathrm{BV}$ ) versus radius of $n+$ type region for different 1D breakdown voltages in non-punch through design.

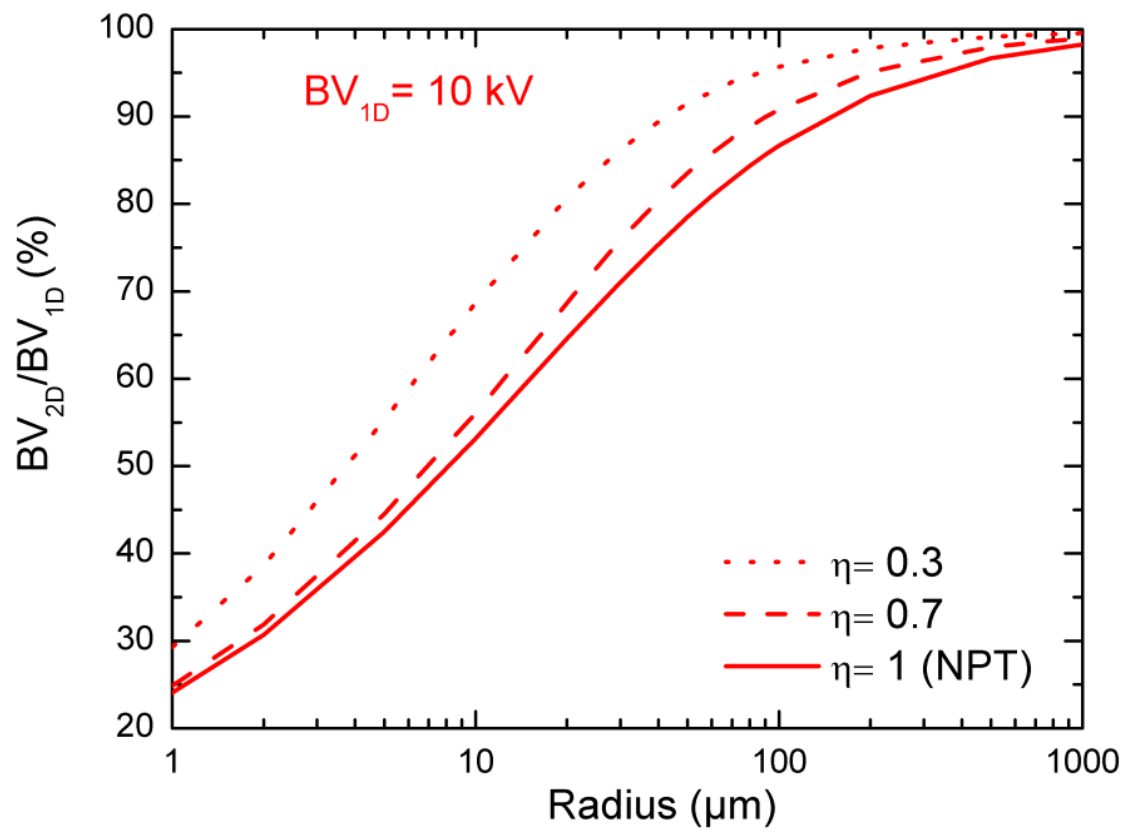

Figure 8: Normalized breakdown voltage (ratio of $2 \mathrm{D} \mathrm{BV}$ by $1 \mathrm{D} \mathrm{BV}$ ) versus radius of $n+$ type region for different reciprocal punch through factors for a $1 D \mathrm{BV}$ of $10 \mathrm{kV}$.

As can be seen on Figure 7, for small breakdown voltages such as $1 \mathrm{kV}$, small radii have a small impact on the reached BV in 2D: only $16 \%$ lower BV than expected for a $1 \mu \mathrm{m}$ radius and since the radius is greater than 10 $\mu \mathrm{m}, \mathrm{BV}$ in $2 \mathrm{D}$ is at least $95 \%$ of $\mathrm{BV}$ in $1 \mathrm{D}$. However, the radius has more influence on the reached normalized $\mathrm{BV}$ while increasing the targeted BV. For instance, for $1 \mathrm{D} \mathrm{BV}$ of $10 \mathrm{kV}$ the $2 \mathrm{D} \mathrm{BV}$ with a radius of $1 \mu \mathrm{m}$ is only $25 \%$ of $1 \mathrm{D} \mathrm{BV}$ and $95 \%$ of $1 \mathrm{D} \mathrm{BV}$ is only reached for radius of $400 \mu \mathrm{m}$. Nevertheless, as a comparison, diamond is far less sensitive to 2D effects than Silicon: e.g. for a $10 \mu \mathrm{m}$ radius the BV 2D of Silicon is only $40 \%$ ( $95 \%$ in diamond case) of the $\mathrm{BV}$ in $1 \mathrm{D}$ of $1 \mathrm{kV}$ and $95 \%$ of the targeted $\mathrm{BV}$ in $1 \mathrm{D}$ is reached for radii larger than $700 \mu \mathrm{m}$. On Figure 8, the effect of the reciprocal PT factors has been investigated at a given BV of $10 \mathrm{kV}$. As can 
been seen, the lower $\eta$ is (i.e. the drift layer and the doping level are smaller), the better it is to get to the BV in $1 \mathrm{D}$ value for small radii. In other word, it is a matter of interest to favor PT design in order to lower as far as possible the $2 \mathrm{D}$ effect on the reached BV. Therefore, punch through design can be beneficial not only to lower the ON state resistance but also to get rid (partially) of 2D effects. This allows us seeing how 2D cylindrical coordinates impact on the electric field lines and so the expected BV. In a real structure 3D effects will also occur and even more lower the targeted BV compared to $1 \mathrm{D}$ prediction. Therefore one must be aware of such limitations as radii between ten and hundred microns are commonly used in the design of such devices. Thus one have to think about favoring small reciprocal PT factors in certain cases and developing efficient JTE.

\section{Toward device performances improvement}

Calculation and prediction exposed in the previous sections will help to design devices with optimum performances. In this part we will show, using data of two experimental devices from literature, how their performances could have been improved by choosing an adapted design in regards of the targeted specifications.

Yang et al. [5] reported Schottky barrier devices able to withstand $8 \mathrm{kV}$ and $12.4 \mathrm{kV}$ (experimental data points are reported on Figure 2) with a drift layer made respectively of $100 \mu \mathrm{m}$ and $300 \mu \mathrm{m}$ of $3 \times 10^{15} \mathrm{~cm}^{-3}$ doped $p$ type diamond. No clear specific ON state resistance value is given in this work. Nevertheless, our algorithm allows computing the electric field profile of such a structure. It appears that for this doping level value, the electric field extends only on the first $45 \mu \mathrm{m}$ of the drift layer in non-punch through design. Thus, more than half (for the 100 $\mu \mathrm{m}$ thick drift layer) of the layer is not useful to withstand the breakdown voltage and will only contribute to decrease ON state performances. By designing this device with a $45 \mu \mathrm{m}$ thick drift layer $(\eta=1)$, the $R_{o n} . S$ value could be theoretically divided by a factor 2 and a factor 6 for the two devices considered hereinbefore. It is even possible to further improve the $\mathrm{ON}$ state performances of these devices by considering a punch through design with the optimum reciprocal PT factor of 0.7 .

On the contrary, some devices are fabricated with very low recirprocal PT factor which also drastically reduces the performances. Traoré et al. [2] fabricated Schottky barrier devices showing a breakdown voltage of 1 $\mathrm{kV}$ and a $R_{o n} . S$ of $6 \mathrm{~m} \Omega . \mathrm{cm}^{2}$ (experimental data reported are also reported on Figure 2 and Figure 3 ). The drift layer of the devices is doped at $3 \times 10^{15} \mathrm{~cm}^{-3}$ and is $1.3 \mu \mathrm{m}$ thick which in fact correspond to a very small reciprocal punch through factor of 0.02 value. As can be seen on Figure 5, the specific ON state resistance increases a lot for very low reciprocal PT factor, degrading the ON state performances. So, considering a PT design with $\eta=0.72$ (or even with $\eta=1$ ) can divide the $\mathrm{ON}$ state resistance by a factor of more than 7 (or 6) as summarized on table 2.

Table 2: Comparison of experimental specifications of Traoré et al. SBD with proposed improved designs.

\begin{tabular}{|c|c|c|c|}
\hline & Experimental design & Improved design 1 & Improved design 2 \\
\hline BV & $1 \mathrm{kV}$ & $1 \mathrm{kV}$ & $1 \mathrm{kV}$ \\
\hline Design type ( $\eta)$ & PT (0.02) & NPT (1) & PT (0.72) \\
\hline Drift layer thickness & $1.3 \mu \mathrm{m}$ & $1.9 \mu \mathrm{m}$ & $1.5 \mu \mathrm{m}$ \\
\hline Doping level & $1.5 \times 10^{15} \mathrm{~cm}^{-3}$ & $1.8 \times 10^{17} \mathrm{~cm}^{-3}$ & $1.5 \times 10^{17} \mathrm{~cm}^{-3}$ \\
\hline$R_{\text {on. }} S$ & $6 \mathrm{~m} \Omega \cdot \mathrm{cm}^{2}$ & $0.9 \mathrm{~m} \Omega . \mathrm{cm}^{2}$ & $0.8 \mathrm{~m} \Omega . \mathrm{cm}^{2}$ \\
\hline
\end{tabular}


The improved designs proposed here are based on keeping the same breakdown voltage than the experimental device, but it is also possible to propose an improvement of the breakdown voltage by keeping the same doping level and increasing the drift layer thickness.

\section{Conclusions}

This study allows us to design the drift layer of doped diamond component in terms of doping level and thickness for a targeted breakdown voltage. The specific ON state resistance of an optimized drift layer can be divided by several orders compared to a non-optimized one for a given breakdown voltage. Results presented in this work allow proposing preliminary design rules to fabricate working and efficient unipolar diamond power devices. However we show that thickness and doping (level and control) required for such structure fabrication are quite challenging for growth in the context of high voltage power devices. Indeed, these two parameters should be well controlled and particularly low doping level (in the order of $10^{15} \mathrm{~cm}^{-3}$ ) with large thickness value (larger than $30 \mu \mathrm{m})$ are necessary for $p$ type drift layer for BV of $10 \mathrm{kV}$. In addition, thanks to a $2 \mathrm{D}$ cylindrical breakdown voltage approach it has been shown that small pattern can lower significantly breakdown voltage, especially for large breakdown voltage design, compared to 1D case, hence the necessity of developing JTE. Such a discrete approach can be very useful to predict performances and to propose design rules, following improvements on diamond avalanche breakdown models. In fact further studies must answer several questions such as: Is the avalanche the only mechanism responsible of breakdown? Which is impact ionization temperature dependence? Could we consider that JTE will be efficient enough to reach breakdown voltage calculated in one dimensional? 


\section{Acknowledgement}

The research leading to these results has been performed within the GreenDiamond project (http://www.greendiamond-project.eu/) and received funding from the European Community's Horizon 2020 Programme (H2020/2014-2020) under grant agreement $\mathrm{n}^{\circ} 640947$.

\section{References}

[1] A. Hiraiwa, H. Kawarada, Figure of merit of diamond power devices based on accurately estimated impact ionization processes, J. Appl. Phys. 114 (2013) 034506. doi:10.1063/1.4816312.

[2] A. Traoré, P. Muret, A. Fiori, D. Eon, E. Gheeraert, J. Pernot, Zr/oxidized diamond interface for high power Schottky diodes, Appl. Phys. Lett. 104 (2014) 052105. doi:10.1063/1.4864060.

[3] P.-N. Volpe, P. Muret, J. Pernot, F. Omnès, T. Teraji, Y. Koide, F. Jomard, D. Planson, P. Brosselard, N. Dheilly, B. Vergne, S. Scharnholz, Extreme dielectric strength in boron doped homoepitaxial diamond, Appl. Phys. Lett. 97 (2010) 223501. doi:doi:10.1063/1.3520140.

[4] H. Umezawa, M. Nagase, Y. Kato, S. Shikata, High temperature application of diamond power device, Diam. Relat. Mater. 24 (2012) 201-205. doi:10.1016/j.diamond.2012.01.011.

[5] J. Yang, W. Huang, T.P. Chow, J.E. Butler, High Quality MPCVD Epitaxial Diamond Film for Power Device Application, in: Symp. B - Prog. Compd. Semicond. Mater. IV - Electron. Optoelectron. Appl., 2004. doi:10.1557/PROC-829-B7.2.

[6] J.E. Butler, M.W. Geis, K.E. Krohn, J. Lawless, S. Deneault, T.M. Lyszczarz, D. Flechtner, R. Wright, Exceptionally high voltage Schottky diamond diodes and low boron doping, Semicond. Sci. Technol. 18 (2003) S67-S71. doi:10.1088/0268-1242/18/3/309.

[7] H. Umezawa, T. Matsumoto, S.-I. Shikata, Diamond Metal Semiconductor Field-Effect Transistor With Breakdown Voltage Over $1.5 \mathrm{kV}$, IEEE Electron Device Lett. 35 (2014) 1112-1114. doi:10.1109/LED.2014.2356191.

[8] P. Gluche, A. Aleksov, A. Vescan, W. Ebert, E. Kohn, Diamond surface-channel FET structure with $200 \mathrm{~V}$ breakdown voltage, IEEE Electron Device Lett. 18 (1997) 547-549. doi:10.1109/55.641441.

[9] S.A.O. Russell, S. Sharabi, A. Tallaire, D.A.J. Moran, Hydrogen-Terminated Diamond Field-Effect Transistors With Cutoff Frequency of $53 \mathrm{GHz}$, IEEE Electron Device Lett. 33 (2012) 1471-1473. doi:10.1109/LED.2012.2210020.

[10] K. Ueda, M. Kasu, High Temperature Operation of Boron-Implanted Diamond Field-Effect Transistors, Jpn. J. Appl. Phys. 49 (2010) 04DF16. doi:10.1143/JJAP.49.04DF16.

[11] K. Hirama, K. Tsuge, S. Sato, T. Tsuno, Y. Jingu, S. Yamauchi, H. Kawarada, High-Performance P-Channel Diamond Metal-Oxide-Semiconductor Field-Effect Transistors on H-Terminated (111) Surface, Appl. Phys. Express. 3 (2010) 044001. doi:10.1143/APEX.3.044001.

[12] K. Hirama, S. Miyamoto, H. Matsudaira, K. Yamada, H. Kawarada, T. Chikyo, H. Koinuma, K. Hasegawa, H. Umezawa, Characterization of diamond metal-insulator-semiconductor field-effect transistors with aluminum oxide gate insulator, Appl. Phys. Lett. 88 (2006) 112117. doi:10.1063/1.2186072.

[13] H. Kawarada, High-Current Metal Oxide Semiconductor Field-Effect Transistors on H-Terminated Diamond Surfaces and Their High-Frequency Operation, Jpn. J. Appl. Phys. 51 (2012) 090111. doi:10.1143/JJAP.51.090111.

[14] H. Kawarada, H. Tsuboi, T. Naruo, T. Yamada, D. Xu, A. Daicho, T. Saito, A. Hiraiwa, C-H surface diamond field effect transistors for high temperature $\left(400{ }^{\circ} \mathrm{C}\right)$ and high voltage $(500 \mathrm{~V})$ operation, Appl. Phys. Lett. 105 (2014) 013510. doi:10.1063/1.4884828.

[15] G. Chicot, A. Maréchal, R. Motte, P. Muret, E. Gheeraert, J. Pernot, Metal oxide semiconductor structure using oxygen-terminated diamond, Appl. Phys. Lett. 102 (2013) 242108. doi:10.1063/1.4811668.

[16] A. Maréchal, M. Aoukar, C. Vallée, C. Rivière, D. Eon, J. Pernot, E. Gheeraert, Energy-band diagram configuration of Al2O3/oxygen-terminated p-diamond metal-oxide-semiconductor, Appl. Phys. Lett. 107 (2015) 141601. doi:10.1063/1.4931123. 
[17] K.K. Kovi, Ö. Vallin, S. Majdi, J. Isberg, Inversion in Metal-Oxide -Semiconductor Capacitors on BoronDoped Diamond, IEEE Electron Device Lett. 36 (2015) 603-605. doi:10.1109/LED.2015.2423971.

[18] N. Ren, K. Sheng, An Analytical Model With 2-D Effects for 4H-SiC Trenched Junction Barrier Schottky Diodes, IEEE Trrans. Electron Devices, 61 (2014).

[19] F.D. Bauer, Compact High-Precision Models for Silicon p-n Step Junction Avalanche-Breakdown Voltages, IEEE Trans. Electron Devices. 58 (2011) 658-663. doi:10.1109/TED.2010.2101077.

[20] A. Hiraiwa, H. Kawarada, Blocking characteristics of diamond junctions with a punch-through design, J. Appl. Phys. 117 (2015) 124503. doi:10.1063/1.4916240.

[21] Y. Kamakura, T. Kotani, K. Konaga, N. Minamitani, G. Wakimura, N. Mori, Ab initio study of avalanche breakdown in diamond for power device applications, in: 2015 IEEE Int. Electron Devices Meet. IEDM, 2015: pp. 5.2.1-5.2.4. doi:10.1109/IEDM.2015.7409632.

[22] D. Planson, P. Brosselard, K. Isoird, M. Lazar, L.V. Phung, C. Raynaud, D. Tournier, Wide bandgap semiconductors for ultra high voltage devices. Design and characterization aspects, in: Semicond. Conf. CAS 2014 Int., 2014: pp. 35-40. doi:10.1109/SMICND.2014.6966383.

[23] H. Arbess, K. Isoird, S. Hamady, M. Zerarka, D. Planson, Original Field Plate to Decrease the Maximum Electric Field Peak for High-Voltage Diamond Schottky Diode, IEEE Trans. Electron Devices. 62 (2015) 2945-2951. doi:10.1109/TED.2015.2456073.

[24] N. Rouger, Electric field distribution and voltage breakdown modeling for any PN junction, COMPEL - Int. J. Comput. Math. Electr. Electron. Eng. 35 (2015) 137-156. doi:10.1108/COMPEL-12-2014-0330.

[25] C. Raynaud, D. Tournier, H. Morel, D. Planson, Comparison of high voltage and high temperature performances of wide bandgap semiconductors for vertical power devices, Diam. Relat. Mater. 19 (2010) 16. doi:10.1016/j.diamond.2009.09.015.

[26] J. He, X. Zhang, Y. Wang, X. Xi, Optimum design of punch-through junction used in bipolar and unipolar high voltage power devices, Solid-State Electron. 46 (2002) 847-851. doi:10.1016/S0038-1101(01)00342-2.

[27] P.-N. Volpe, J. Pernot, P. Muret, F. Omnès, High hole mobility in boron doped diamond for power device applications, Appl. Phys. Lett. 94 (2009) 092102-092102-3. doi:doi:10.1063/1.3086397.

[28] A. Maréchal, N. Rouger, J.-C. Crébier, J. Pernot, S. Koizumi, T. Teraji, E. Gheeraert, Model implementation towards the prediction of $\mathrm{J}(\mathrm{V})$ characteristics in diamond bipolar device simulations, Diam. Relat. Mater. 43 (2014) 34-42. doi:10.1016/j.diamond.2014.01.009.

[29] J.-P. Lagrange, A. Deneuville, E. Gheeraert, Activation energy in low compensated homoepitaxial borondoped diamond films, Diam. Relat. Mater. 7 (1998) 1390-1393. doi:16/S0925-9635(98)00225-8.

[30] T. Iwasaki, Y. Hoshino, K. Tsuzuki, H. Kato, T. Makino, M. Ogura, D. Takeuchi, T. Matsumoto, H. Okushi, S. Yamasaki, M. Hatano, Diamond Junction Field-Effect Transistors with Selectively Grown $\mathrm{n}^{+\$}$-Side Gates, Appl. Phys. Express. 5 (2012) 091301. doi:10.1143/APEX.5.091301.

[31] H. Umezawa, Y. Kato, S. Shikata, $1 \Omega$ On-Resistance Diamond Vertical-Schottky Barrier Diode Operated at $250{ }^{\circ} \mathrm{C}$, Appl. Phys. Express. 6 (2013) 011302. doi:10.7567/APEX.6.011302.

[32] H. Umezawa, N. Tatsumi, S.I. Shikata, K. Ikeda, R. Kumaresan, Increase in Reverse Operation Limit by Barrier Height Control of Diamond Schottky Barrier Diode, IEEE Electron Device Lett. 30 (2009) 960-962. doi:10.1109/LED.2009.2026439.

[33] M.I. Landstrass, M.A. Plano, M.A. Moreno, S. McWilliams, L.S. Pan, D.R. Kania, S. Han, Device properties of homoepitaxially grown diamond, Diam. Relat. Mater. 2 (1993) 1033-1037. doi:10.1016/09259635(93)90269-8.

[34] A. Vescan, I. Daumiller, P. Gluche, W. Ebert, E. Kohn, High temperature, high voltage operation of diamond Schottky diode, Diam. Relat. Mater. 7 (1998) 581-584. doi:10.1016/S0925-9635(97)00200-8.

[28] T. Kotani, M. van Schilfgaarde, Impact ionization rates for Si, GaAs, InAs, ZnS, and GaN in the GW approximation, Phys. Rev. B. 81 (2010) 125201. doi:10.1103/PhysRevB.81.125201.

[36] H. Niwa, J. Suda, T. Kimoto, Impact Ionization Coefficients in 4H-SiC Toward Ultrahigh-Voltage Power Devices, IEEE Trans. Electron Devices. 62 (2015) 3326-3333. doi:10.1109/TED.2015.2466445.

[37] I.C. Kizilyalli, T. Prunty, O. Aktas, 4-kV and 2.8- -cm2 Vertical GaN p-n Diodes With Low Leakage Currents, IEEE Electron Device Lett. 36 (2015) 1073-1075. doi:10.1109/LED.2015.2474817.

[38] N. Kaji, H. Niwa, J. Suda, T. Kimoto, Ultrahigh-Voltage SiC PiN Diodes with an Improved Junction Termination Extension Structure and Enhanced Carrier Lifetime, Jpn. J. Appl. Phys. 52 (2013) 070204. doi:10.7567/JJAP.52.070204. 